\title{
Plasma transport properties in the presence of MHD modes studied by ECE at TEXTOR*
}

\author{
V.S. Udintsev ${ }^{\mathrm{a}}$, B.Ph. van Milligen ${ }^{1}$, F.C. Schüller, \\ A. Krämer-Flecken², A.J.H. Donné, J.C. van Gorkom, \\ C.W. Domier ${ }^{3}$ and the TEXTOR-team ${ }^{2}$
}

\author{
FOM-Instituut voor Plasmafysica Rijnhuizen, Association EURATOM-FOM, PO Box 1207, \\ 3430 BE Nieuwegein, The Netherlands ${ }^{\mathrm{b}}$ \\ ${ }^{1}$ Asociación EURATOM-CIEMAT para Fusión, Madrid, Spain \\ ${ }^{2}$ Institut für Plasmaphysik, Forschungszentrum Jülich GmbH, Jülich, Germany ${ }^{\mathrm{b}}$ \\ ${ }^{3}$ Department of Applied Science, University of California at Davis, USA \\ E-mail: udintsev@drfc.cad.cea.fr
}

Received 23 December 2002, accepted for publication 5 September 2003

Published 3 November 2003

Online at stacks.iop.org/NF/43/1424

\begin{abstract}
The influence of large- and small-scale magnetohydrodynamic (MHD) modes on plasma transport properties has been studied. Poloidal and toroidal reconstruction of large $m=2$ modes and their evolution in time have been analysed, as well as their electron temperature profiles and heat transport properties with respect to the background plasma. The confinement is improved within the magnetic island, compared to the background plasma as evidenced by a secondary temperature maximum at the centre of the island. The perpendicular heat diffusivity inside the island is estimated to be of the order of $0.01 \mathrm{~m}^{2} \mathrm{~s}^{-1}$, which is close to the neoclassical value. By cross-correlation of different electron cyclotron emission (ECE) channels, temperature fluctuations in the presence of MHD modes are investigated. For $m=2$ islands, they are found to be different for the X-and the O-points. Evidence of a small mode with higher $m$ number between the $q=1$ and 2 radii is given.
\end{abstract}

PACS numbers: 52.35.Py, 52.55.Fa, 52.35.Tn, 52.70.Gw

\section{Introduction}

Magnetohydrodynamic (MHD) modes and their influence on the transport properties of tokamak plasmas are important research themes at TEXTOR [1,2]. The evolution of the electron temperature distributions of the islands in relation to that of the background plasma is studied by means of fast electron cyclotron emission (ECE) diagnostics. The ECE diagnostic set-up at TEXTOR tokamak makes it possible to analyse the temperature gradients inside the island in detail. These observation help to understand the flow of the heat flux from the plasma core around and inside the island. At TEXTOR, the temperature and density profiles are essentially flat inside the island. Moreover, under certain conditions, when radiation losses inside large $m / n=2 / 1$ mode are not dominant, and its width increases linearly, a secondary

* This is an extended version of the paper presented at the 12th Joint Workshop on ECE and ECRH (Aix-en-Provence, 13-16 May 2002).

a Author to whom any correspondence should be addressed.

b Partners in the Trilateral Euregio Cluster. temperature and density peaking are observed inside the island [1]. This is an indication that the confinement inside the island is improved, compared to the background plasma. One should note, however, that the secondary density peaking is much more pronounced than the secondary temperature peaking. In this paper, the heat diffusivities along and across the field lines inside the large $m / n=2 / 1$ island in TEXTOR are analysed using the extended Rutherford model [3] and the resistance network model [4].

Investigation of the temperature fluctuations in the presence of MHD modes has been made by means of crosscorrelation between different ECE channels. Since a dedicated ECE correlation diagnostic will only become available in the near future, first analysis has been made using different channels of the ECE-imaging system (see section 2). With some assumptions, cross-correlation has been done between spatially separated ECE diagnostics, such as ECE-imaging and the radial ECE systems. Temperature fluctuations are found to be different for the O- and X-points of the $m=2$ island. Another striking result is an observation by ECE of the 


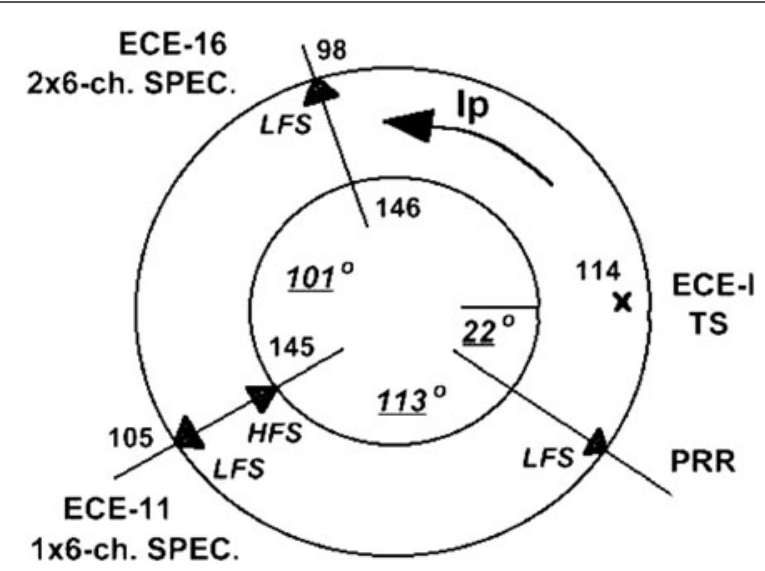

Figure 1. A schematic (top) view of the TEXTOR ECE diagnostics: the 11-channel radiometer (ECE-11) and 16-channel system (ECE-16) are located at approximately $135^{\circ}$ and $236^{\circ}$, respectively, with respect to the location of the ECE-imaging (ECE-I). Also the locations of the pulsed radar reflectometer (PRR) and the Thomson scattering (TS) system are indicated. The toroidal separation in degrees between different diagnostic ports is shown, as are the frequencies of the various ECE systems and the locations of their antennae.

existence of small modes with higher $m$ number in the $q=1$ and 2 vicinities, that has been earlier observed on the density evolution by the pulsed radar reflectometer $[5,6]$.

This paper is organized as follows. In section 2, the various diagnostics used for the analysis are described. Transport properties of plasma in presence of large MHD modes, as well as some aspects of the island rotation are discussed in section 3 . The results of the temperature fluctuation measurements, associated with large and small modes, are presented in section 4. Finally, a conclusion is given in section 5 .

\section{Diagnostic set-up and machine parameters}

The set of ECE (second-third harmonic, X-mode) diagnostics at the TEXTOR tokamak $\left(R_{0}=1.75 \mathrm{~m}, a=0.46 \mathrm{~m}\right.$, $B_{\mathrm{t}}<2.9 \mathrm{~T}$ ) consists of five standard heterodyne radial systems and one vertical oriented imaging system. The diagnostics used for this paper include a radial set consisting of three six-channel spectrometers (104-114, 125-130, 133-148 GHz, sampling rate up to $50 \mathrm{kHz}$ ) and a 16-channel heterodyne ECE radiometer $(96-146 \mathrm{GHz}, 2 \mathrm{MHz})$ all using the same low field side (LFS) antenna, and 11-channel radiometer (105-145 GHz, $25 \mathrm{kHz}$ ) with a combined LFS/HFS antenna [7]. The 16-channel ECE-imaging system $(400 \mathrm{kHz}$, spatial resolution of $1.3 \mathrm{~cm}$ ) measures along a vertical chord at $114 \mathrm{GHz}$, ranging from -0.1 to $0.1 \mathrm{~m}$ with respect to the equatorial plane $[8,9]$.

The 11-channel heterodyne radiometer is absolutely calibrated by means of a hot-cold source. All other ECE diagnostics are cross-calibrated with respect to the 11-channel radiometer, or to the high-resolution Thomson scattering system $[10,11]$.

To study the electron density profile evolution as well as coherent and broadband density fluctuations, a pulsed radar reflectometer is being used [12]. It is based on the measurement of the time-of-flight of short ( $\sim 1 \mathrm{~ns})$ microwave pulses that are reflected in the plasma at density layers where the microwave frequency equals the local plasma frequency (for the O-mode polarization).

The Doppler-shift of the $\mathrm{C}$ vi line measured by charge exchange recombination spectroscopy (CXRS) allows to reconstruct toroidal ion rotation profiles. This system requires a co-neutral beam injector (NBI-1) to be operational. A VUV/XUV spectrometer is used to study impurities in plasma.

The location of various diagnostics at TEXTOR is shown in figure 1.

\section{Transport properties of plasma in presence of large MHD modes}

Large MHD modes at TEXTOR are usually created by a fast density ramp up with a slower current ramp up in the early phase of the discharge [2]. To increase the mode width and to reduce and flatten the toroidal plasma (counter-)rotation profile, co-neutral beam injection (co-NBI) of a few hundreds of kilowatt is being applied. With reduced rotation, wall stabilization is also reduced, which results in general in larger island width. Counter neutral beam injection (counter-NBI) (at lower than co-NBI power) is being used to prevent the mode from wall locking and, thus, from a disruption. In figure 2, a typical example of a discharge with a large $m=2$ mode is shown. The current ramp up is continued up to $1 \mathrm{~s}$, and the line averaged electron density has reached its maximum of $2.2 \times 10^{19} \mathrm{~m}^{-3}$ at $\sim 0.9 \mathrm{~s}$. The toroidal magnetic field $B_{\mathrm{t}}$ was $2.4 \mathrm{~T}$ at the geometric radius of the vessel $R_{0}=1.75 \mathrm{~m}$. No additional gas puff has been performed in this shot.

\subsection{The $q=2$ radial position and $m / n=2 / 1$ island width calculations}

Using the different ECE diagnostics, the evolution of the electron temperature profiles has been studied for the rotating $m / n=2 / 1$ island. In order to estimate the island width properly, the $q=2$ surface position should be determined correctly. Since most of the magnetic diagnostics were not available for this series of shots, the $q=2$ position can be either determined from the inversion of the ECE oscillations between different channels, or calculated from the current density profile, which is derived from the temperature profile assuming Spitzer resistivity.

For the same shot as in figure 2, the temporal evolution of the temperature profiles through the $\mathrm{O}$ - and the $\mathrm{X}$-points of the island has been studied. Using a coordinate transformation and taking the total plasma displacement of $\sim 9 \mathrm{~cm}$ into account (which is a combination of a programmed radial plasma displacement and the Shafranov shift), it is possible to have the full temperature profiles using the data from all ECE diagnostics, even though they measure at different toroidal and poloidal positions. Figure 3 shows the evolution of the temperature profiles inside/outside the $m / n=2 / 1$ island at the LFS at six points in time. The temperature flattening inside the island is clearly seen. The $q=2$ position is determined to be at the crossing of the profiles through the $\mathrm{O}$ - and the X-points of the island. One should note that 

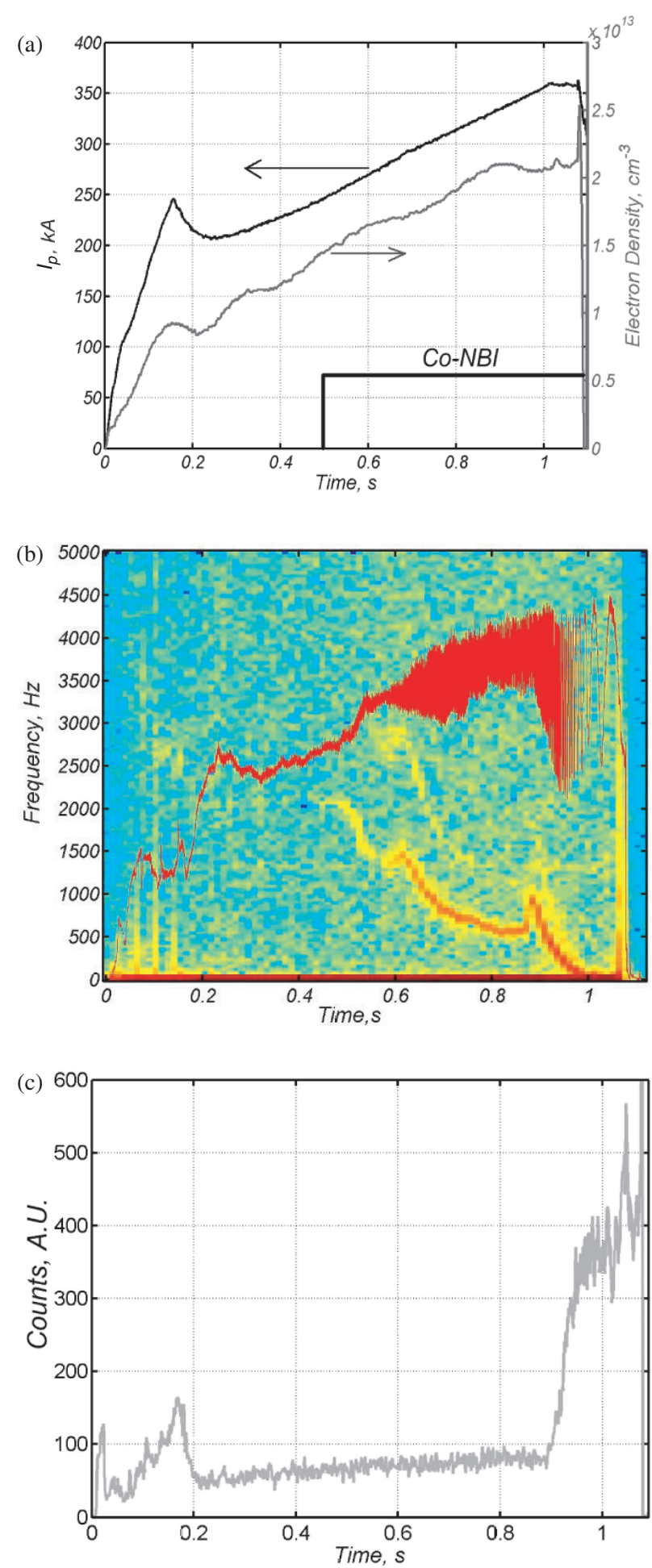

Figure 2. Example of a discharge where a large $m=2$ mode has been created. For this shot, the magnetic field $B_{\mathrm{t}}=2.4 \mathrm{~T}$. Co-NB $(\sim 160 \mathrm{~kW})$ injection is applied at $0.5 \mathrm{~s}$. Evolution of the electron density and plasma current is shown in $(a)$. Spectrogram $(b)$ shows that the frequency of the mode is slowing down from 1.5 to $0.5 \mathrm{kHz}$, then spinning up to $1 \mathrm{kHz}$ at $\sim 0.88 \mathrm{~s}$ during a very short time interval, and slowing down again finally leading to a disruption. At $\sim 0.88 \mathrm{~s}$, an increase of the $\mathrm{C}$ IV intensity $(c)$ is observed by the VUV/XUV spectrometer. The mode activity is clearly seen on the $115 \mathrm{GHz}$ ECE channel at the time between 0.45 and $1.07 \mathrm{~s}(b)$. the plasma current is still in the ramp up phase until $\sim 1 \mathrm{~s}$, so the $q=2$ surface is shifting outwards. It can also be seen that the asymmetry of the island is increasing, as the island grows. Around $t \sim 1 \mathrm{~s}$, the $q=2$ surface moves out of sight of the $112 \mathrm{GHz}$ ECE channel at $R=2.11 \mathrm{~m}$, and there are only two ECE channels located outside $q=2$ at $R=2.17 \mathrm{~m}(109 \mathrm{GHz})$ and $R=2.205 \mathrm{~m}(107 \mathrm{GHz})$. Under the assumption that outside and close to the island separatrix the electron temperature is a flux function [13], the $q=2$ position has been calculated for this time using the following relations [14]:

$$
\begin{aligned}
\Delta r & =\frac{\Delta T_{\mathrm{e}}}{\mathrm{d} T_{\mathrm{e}} / \mathrm{d} r}, \\
R_{q=2} & =R_{114}+\Delta r,
\end{aligned}
$$

where $\Delta T_{\mathrm{e}}$ is the difference between temperatures at the $\mathrm{X}$ - and O-points of the island at $R_{114}=2.07 \mathrm{~m}$ (as measured by the $114 \mathrm{GHz}$ ECE channel), $\mathrm{d} T_{\mathrm{e}} / \mathrm{d} r$ is the temperature gradient at the $\mathrm{X}$-point calculated for the same $R, \Delta r$ is a displacement of the flux surfaces in the neighbourhood of the separatrix, which is proportional to the change of the island width $w, R_{114}$ is the location of the $114 \mathrm{GHz}$ channel $\left(R_{114}-R_{0}=0.32 \mathrm{~m}\right)$. If the island is radially symmetrical with respect to the $q=2, \Delta r$ can be described as the island half-width $w / 2$. This can be seen in figure 3 for the times before $0.85 \mathrm{~s}$. Close to the disruption at $1.07 \mathrm{~s}$, however, the asymmetry between the core-facing and the wall-facing separatrices is very large, and $\Delta r$ almost equals to the island width $w$.

Another method to determine the $q=2$ radial position is to calculate it from the toroidal current density profile evolution. For the plasma parameters in this shot, neoclassical corrections to Spitzer's resistivity $[15,16]$ can be neglected. Then, the radial profile of $q$ can be calculated as follows:

$$
q(\rho)=\frac{\rho^{2} B_{\mathrm{t}}\left(R_{0}\right)}{\mu_{0} R_{0} k_{\mathrm{s}} \int_{0}^{\rho} \rho^{\prime} T_{\mathrm{e}}^{3 / 2}\left(\rho^{\prime}\right) \mathrm{d} \rho^{\prime}} .
$$

Here, $k_{\mathrm{s}}$ is a constant that depends on the total plasma current $I_{\mathrm{pa}}$, ion effective plasma charge $Z_{\mathrm{eff}}$ and the electron temperature profile $T_{\mathrm{e}}(\rho), \rho=[r-\Delta(\rho)] / a=$ $\left[\left(R-R_{0}\right)-\Delta(\rho)\right] / a$ is the dimensionless flux surface coordinate that depends on the plasma displacement of each flux surface $\Delta(\rho)$. For the NBI-heated discharges at TEXTOR, $Z_{\text {eff }}$ typically varies from 2.5 to 3.5 [17].

In figure $4(a)$, the dimensionless island width $w / a$ and displacement $\Delta r / a$ are shown for the same shot as in figures 2 and 3. The width of the island is assumed to be equal to the radial size of the flat spot at the O-point (as seen in figure 3). Using equation (3.1.1), the $\Delta r$ has been calculated. The error bars are defined from the spatial resolution for each channel and from the radial separation between the channels. Figure 4(b) shows the evolution in time of the $q=2$ position as determined from the profiles in figure 3 and calculations using equation (3.1.3). In figure 4(c), the calculated radial $q$ profile is shown for $\sim 0.97 \mathrm{~s}$. It has been found from the calculation of current density profiles with both Spitzer and neoclassical resistivities that less than $5 \%$ of the total toroidal plasma current is flowing outside $q=2$. Then, one can assume that the radial position of the $q=2$ surface does not depend too strongly on the shape of the current density profile. For this particular shot, no sawtooth activity, as well as no clear 
MHD modes on plasma transport properties
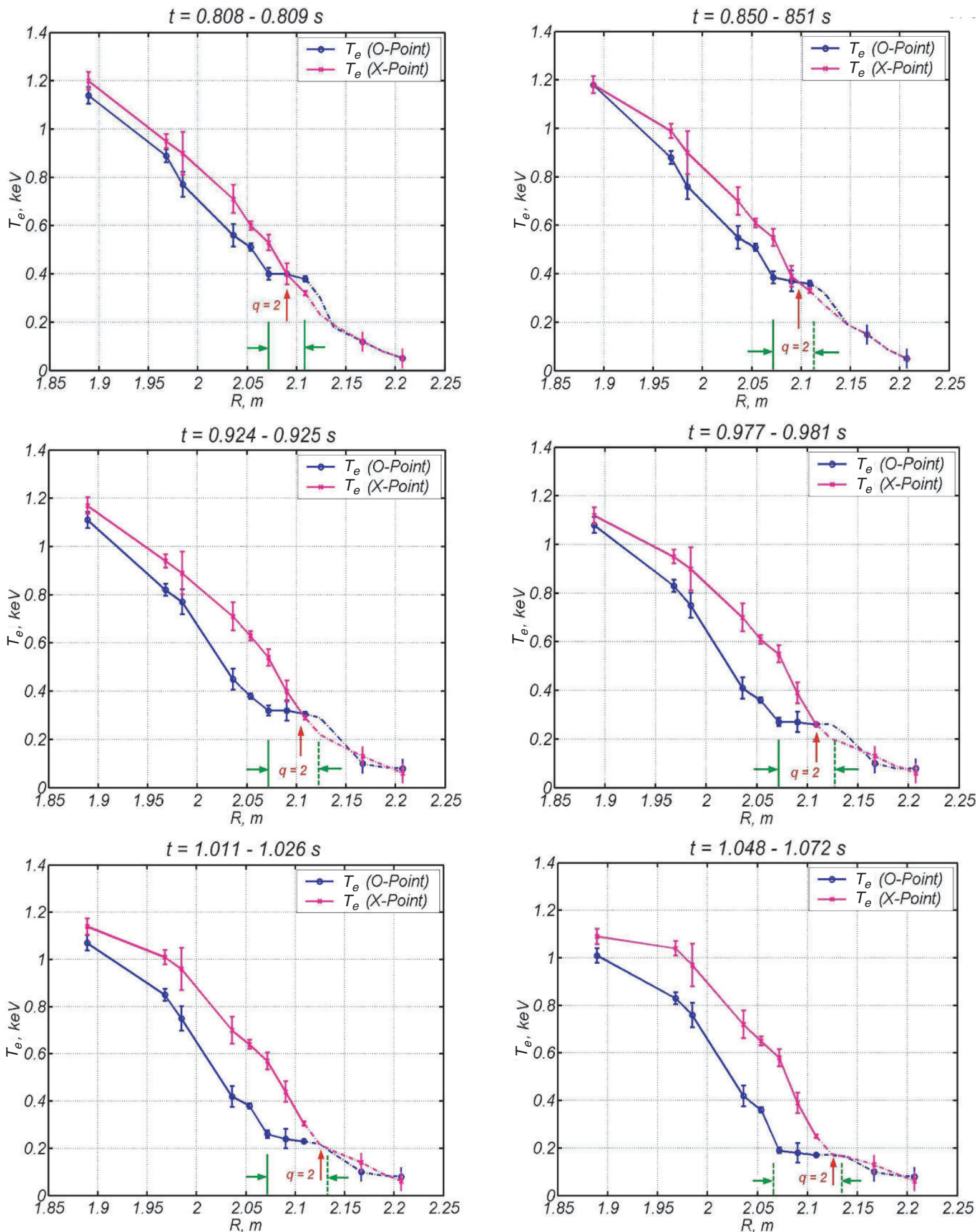

Figure 3. Temporal evolution of temperature profiles through $\mathrm{O}$ (blue circles) and $\mathrm{X}$ (magenta crosses) points of the large $m / n=2 / 1$ island at the LFS. As the mode grows, its asymmetry with respect to the $q=2$ position becomes more pronounced. At $\sim 1.05 \mathrm{~s}$, the total island width (as shown by green arrows) is almost equal to its displacement $\Delta r$. Dashed lines are extrapolated temperature profiles, since there are only a very few ECE channels located outside $q=2$. Between $R=2.170-2.205 \mathrm{~m}$, also a small $\mathrm{m} / n=3 / 1$ mode activity has been detected.

$m / n=1 / 1$ mode, have been observed, which suggest $q_{0}$ to be greater than 1 . The value of $q_{\mathrm{a}}$ depends on the total plasma current and changes from 4.6 for $I_{\mathrm{pa}}=315 \mathrm{kA}$ at $0.8 \mathrm{~s}$ to 4.03 for $I_{\mathrm{pa}}=360 \mathrm{kA}$ at $1 \mathrm{~s}$. Some values calculated for $\sim 0.97 \mathrm{~s}$ are shown in table 1. It can be seen that the agreement between measured and calculated evolution of the $q=2$ position is quite good; in the order of the spatial resolution of the ECE diagnostics. 

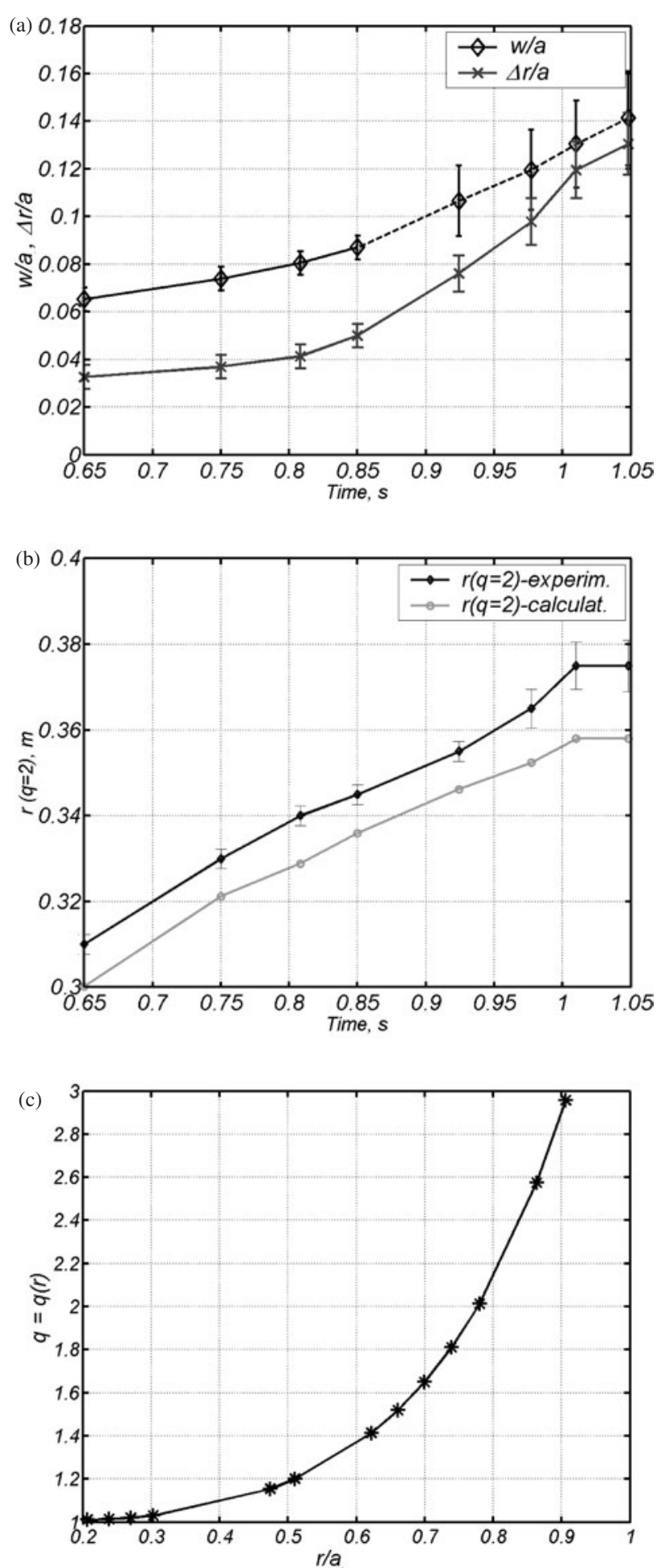

Figure 4. The dimensionless width $w$ and displacement $\Delta r$ of the large $m / n=2 / 1$ island ( $a$ ) and the $q=2$ position $(b)$ as functions of time. As can be seen from plot $(a)$, the island width grows linearly up to $\sim 0.8 \mathrm{~s}$, and then its growth rate increases markedly. The calculated radial $q$ profile at $\sim 0.97 \mathrm{~s}$ is shown in $(c)$.

\subsection{Transport properties of plasma in presence of the large $m / n=2 / 1$ island}

As has been reported earlier in [1], secondary temperature and density peaking inside the $m / n=2 / 1$ island has
Table 1. Some parameters at $q=2$ position $\left(R_{q=2} \approx 2.11 \mathrm{~m}\right)$ for the time of $\sim 0.97 \mathrm{~s}$ (see figure 3 ).

\begin{tabular}{llllll}
\hline $\begin{array}{l}T_{\mathrm{e}} \\
(\mathrm{eV})\end{array}$ & $\begin{array}{l}n_{\mathrm{e}} \\
\left(\mathrm{m}^{-3}\right)\end{array}$ & $\begin{array}{l}\tau_{\mathrm{e}} \\
(\mathrm{s})\end{array}$ & $\begin{array}{l}\eta_{\mathrm{Sp}} \\
(\Omega \mathrm{m})\end{array}$ & $\begin{array}{l}j \\
\left(\mathrm{kA} \mathrm{m}^{-2}\right)\end{array}$ & $\begin{array}{l}v_{\mathrm{e}}^{\text {thermal }} \\
\left(\mathrm{m} \mathrm{s}^{-1}\right)\end{array}$ \\
\hline 260 & $2 \times 10^{19}$ & $2 \times 10^{-6}$ & $5 \times 10^{-7}$ & 100 & $2 \times 10^{7}$ \\
\hline
\end{tabular}

been observed indicating an improved confinement inside the island. Although the secondary density peaking is almost always present, as evidenced by the pulsed radar reflectometer measurements, the secondary temperature peaking appearance depends strongly on the island width and asymmetry. Figures $5(a)$ and $(b)$ show the evolution of the pulsed radar reflectometer signal at $47 \mathrm{GHz}$ and HFS ECE channels at 140 and $145 \mathrm{GHz}$. For this shot, $B_{\mathrm{t}}=2.25 \mathrm{~T}$ at the plasma centre, and $n_{\mathrm{e}}=2.2 \times 10^{19} \mathrm{~m}^{-3}$ at the $q=2$ radius. The secondary temperature peaking of $30 \mathrm{eV}$, compare to the temperature at the island's separatrix, is observed by the ECE channel. A secondary density and temperature peaking inside the island has been observed before by the Thomson scattering measurements at RTP [14] and TEXTOR [18, 19]. An example is shown in figure $5(c)$.

For the same shot as shown in figures $2-4$ and parameters given in table 1 , the transport properties of the TEXTOR plasma in the presence of the large islands have been studied using the extended Rutherford model $[3,14]$ and the resistance network analogy model described in [4].

In this TEXTOR shot, the total temperature flattening inside the island at the LFS is clearly seen (see figure 3). The resistance network model has been applied for $t=0.97 \mathrm{~s}$. The ratio between parallel and perpendicular transport times inside the island can be expressed as follows:

$$
\frac{\tau_{\|}}{\tau_{\perp}}=\left(\frac{m \pi R q^{*}}{w}\right)^{2} \frac{\chi_{\perp}}{\chi_{\|}} .
$$

Here, $q^{*}$ is the safety factor related to the field line helicity around the magnetic axis of the island just inside the separatrix. In general, $q^{*}$ is a function of the current density profile inside the island:

$$
q^{*}=\frac{8 \pi r^{2} B_{\mathrm{t}}\left(R_{0}\right)}{\mu_{0} m^{3} \delta I}
$$

where $\delta I$ is an incremental current associated with the $\mathrm{O}$-point of the island. From [20], the island width can be expressed in terms of the radial magnetic field perturbation at the rational surface $\tilde{B}_{r}$ :

$$
w=4 \sqrt{\frac{\rho q}{m B_{\mathrm{p}} \partial q / \partial \rho}} \sqrt{\tilde{B}_{r}}
$$

with all the quantities taken at the rational surface. From $\tilde{B}_{r}$, the amplitude of the perturbed component of the poloidal field can be deduced for the given $m$ and $n$ mode numbers, and the incremental current $\delta I$ can be calculated. Unfortunately, no useful magnetic diagnostics were available for this shot programme to measure the perturbed poloidal magnetic field. Nevertheless, a very rough approximation can be made using equation (3.2.3) and following the method for the $\delta I$ calculation described in [4]. Then, $\tilde{B}_{r} \sim 2 \times 10^{-3} \mathrm{~T}$, and 

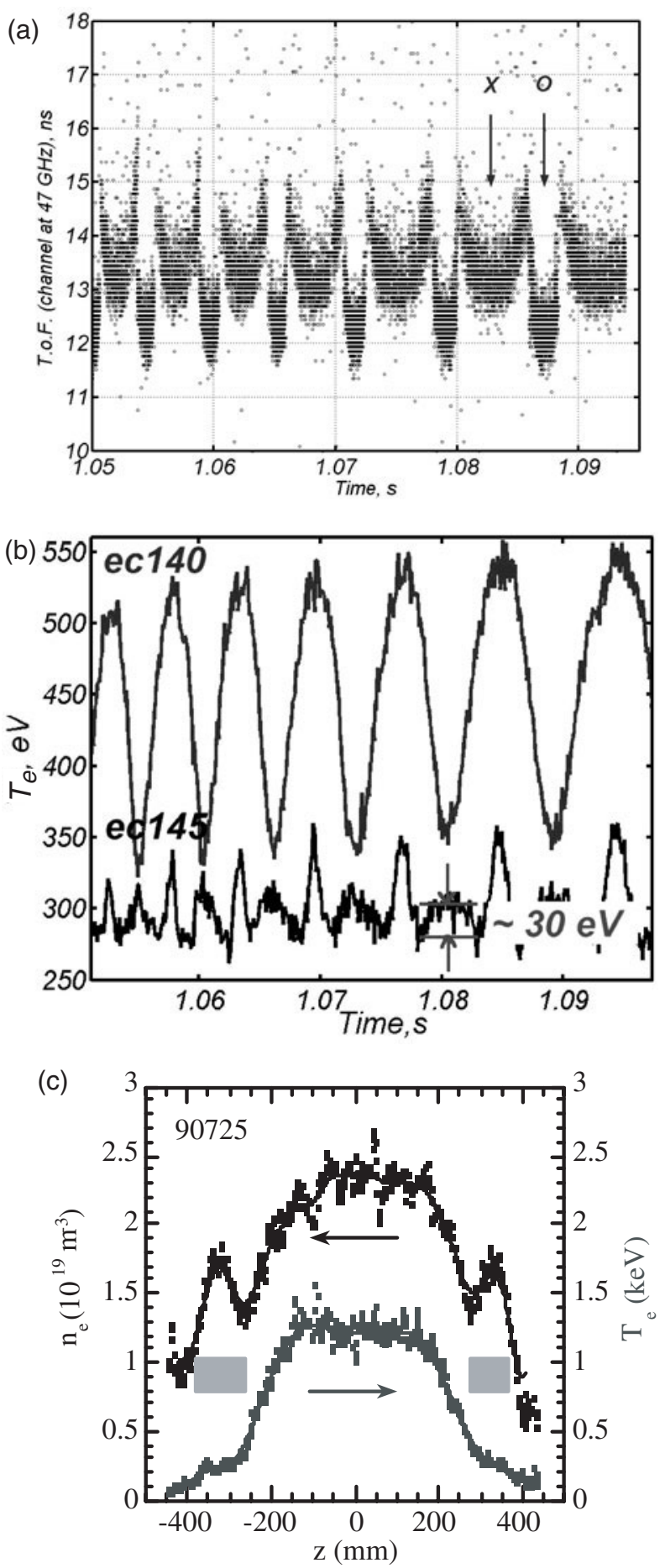

Figure 5. Secondary density and temperature peaking inside the large $m / n=2 / 1$ island as evidenced by the pulsed radar reflectometer at the LFS and ECE at the HFS $(a)$ and $(b)$. For this shot, the island width is estimated to be about $4-5 \mathrm{~cm}$. The island is symmetrical at the HFS, with respect to the $q=2$ position. The Thomson scattering profiles $(c)$ confirm the secondary density peaking and temperature flattening inside the island. $(c)$ is taken from [19] with kind permission of the authors.

$\delta I$ is estimated to be in the order of a few kiloampere. Equation (3.2.2) can be written in the following form:

$$
q^{*}=32 \pi \frac{\rho q^{2}}{m^{3} w^{2} \partial q / \partial \rho} .
$$

For the island width of 5.5-6.0 $\mathrm{cm}$ and $\partial q / \partial \rho \approx 12 \mathrm{~m}^{-1}$, the $q^{*}$ value calculated by using equation $(3.2 .2 a)$ is found to be about 350 . The calculated $q^{*}$ value depends strongly on the island width $w$ and radial position $\rho$, which are determined from the measurements. Then, the error bar for $q^{*}$ can be quite considerable.

For the parameters given in table 1, the parallel heat diffusivity $\chi_{\|} \sim v_{\mathrm{e}}^{2} \tau_{\mathrm{e}}$ is calculated to be $\sim 6 \times 10^{8} \mathrm{~m}^{2} \mathrm{~s}^{-1}$. The temperature flattening inside the island is very pronounced and the difference between temperatures in the $\mathrm{O}$-point and the background plasma at the same radius is high (figure 3), thus, the ratio of $\tau_{\|} / \tau_{\perp}$ much smaller than 1 is expected [4]. From equation (3.2.1), one can estimate $\chi_{\perp}$ to be about $0.13 \tau_{\|} / \tau_{\perp}$, so the expected value approaches the neoclassical.

Similar conclusions can be drawn if one applies the extended Rutherford model $[3,14]$ to TEXTOR plasmas. The island growth can be written as follows (with all quantities taken at the resonant surface):

$$
\frac{\mathrm{d} w}{\mathrm{~d} t}=1.22 \frac{\eta}{\mu_{0}} \Delta^{\prime}-\left(\frac{0.9 \eta j q}{B_{\mathrm{p}} \partial q / \partial \rho}\right) \frac{\tilde{P}_{\mathrm{T}}}{\chi_{\perp} T_{\mathrm{e}}} .
$$

Here, $\Delta^{\prime}$ is the stability factor depending on the local averaged $\nabla j, \tilde{P}_{\mathrm{T}}$ is the total net power density per particle in the island, $j$ is the current density. If the radiation losses are not important, the second term in equation (3.2.4) can be neglected. The island grows linearly during the time interval of $0.65-0.8 \mathrm{~s}$ (see figure 4 ), and $\Delta^{\prime}$ is calculated to be $\sim 0.5$. A faster growth starts after $0.85 \mathrm{~s}$. As $\Delta^{\prime}$ does not change very much, it means that $\tilde{P}_{\mathrm{T}}$ becomes negative due to the increasing radiation losses. It should be noted that at this time the enhancement in the $C$ Iv line is observed by the VUV/XUV spectrometer (see figure 2). This is an indication that the impurity level has rapidly increased in a very thin layer (of $2-3 \mathrm{~cm}$ ) close to the wall, and the plasma edge has started to cool down. Simultaneously, the temperature inside the island drops from $390 \mathrm{eV}$ at $0.8 \mathrm{~s}$ down to $190 \mathrm{eV}$ prior to the disruption, yielding an increase in the radiation losses inside the island. Integration and solution of equation (3.2.4) gives: $\tilde{P}_{\mathrm{T}} / \chi_{\perp} \approx-1.1 \times 10^{5} \mathrm{eV} \mathrm{m}^{-2}$. Since temperature inside the island drops over $200 \mathrm{eV}$ from 0.8 to $1.07 \mathrm{~s}, \tilde{P}_{\mathrm{T}}$ is found to be of the order of $1000 \mathrm{eV} \mathrm{s}^{-1}$ and, therefore, $\chi_{\perp} \sim 0.01 \mathrm{~m}^{2} \mathrm{~s}^{-1}$. This is in agreement with the result obtained using equation (3.2.1), and much less than the global heat diffusivity of about $1.5 \mathrm{~m}^{2} \mathrm{~s}^{-1}$ following from the energy confinement time $\tau_{E}$ of $30-40 \mathrm{~ms}$ for the NBI-heated plasmas at TEXTOR.

\subsection{Some aspects of modes rotation in TEXTOR plasma}

The rotation of the large MHD modes in TEXTOR has been extensively studied in the past [1]. However, using the unique feature of the ECE-imaging system to observe a vertical line of sight in the vicinity of the $q=2$ surface at the LFS, it is possible to study the island rotation in the poloidal direction. A typical example is shown in figure $6(a)$, where the large $m / n=2 / 1$ mode has inverted its toroidal rotation direction at $\sim 0.565 \mathrm{~s}$ due to the co-NB injection, and locks to the wall at $\sim 0.63 \mathrm{~s}$. As can be seen from the contour plots in figures $6(d)$ and $(e)$, the total island rotation velocity projected on the poloidal direction is $\sim 1100-1200 \mathrm{~m} \mathrm{~s}^{-1}$ and $300-350 \mathrm{~m} \mathrm{~s}^{-1}$ before and after the rotation inversion, respectively. Taking into account that the magnetic island is a helical structure rotating with the plasma, 
(a)

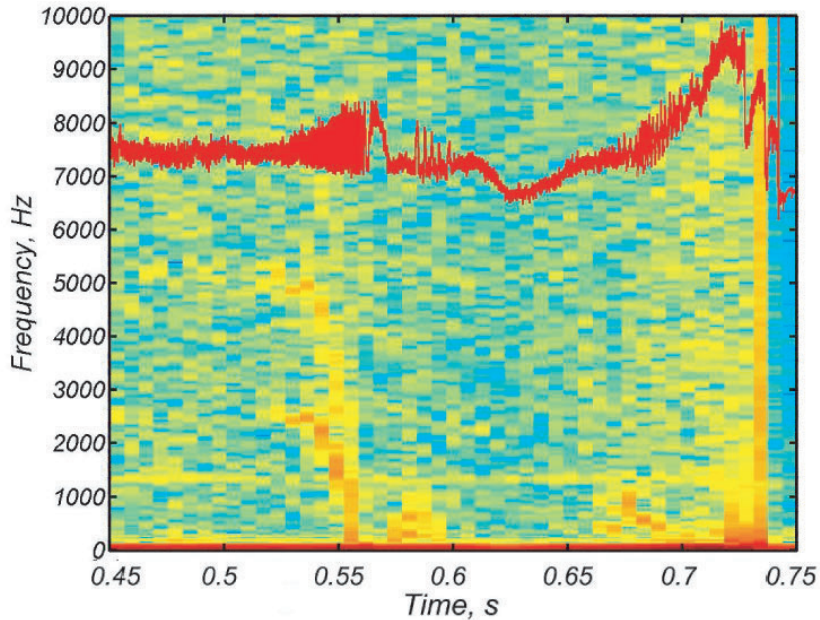

(b)

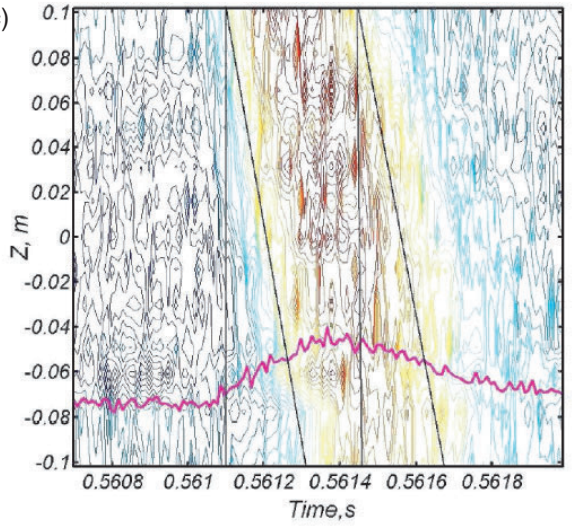

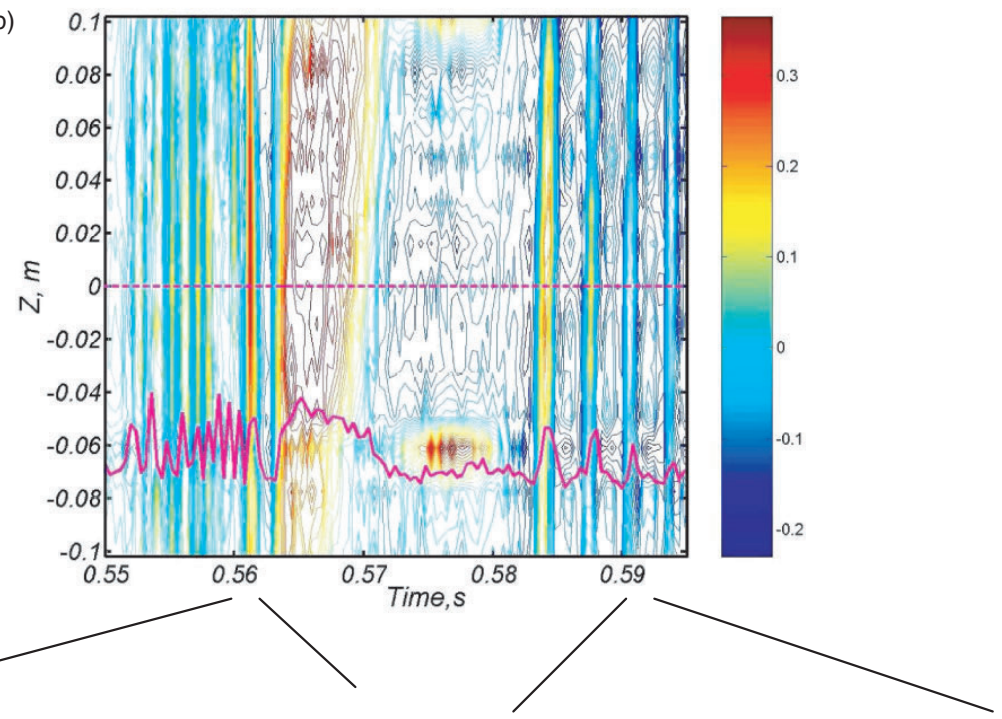

(d)

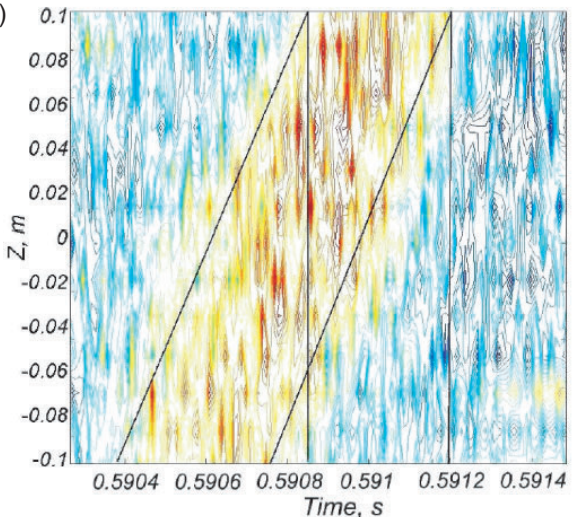

Figure 6. (a) A large $m / n=2 / 1$ mode and its spectrogram as monitored by the ECE-imaging system. The rotation inversion occurs at $\sim 0.565 \mathrm{~s} ;(b)-(d)$ contour plot of the normalized temperature measured by ECE-imaging. The position of the central channel is shown by the dotted line. The hot spot at $z=-0.06$ is due to the noise of the channel at this position. Lines in $(c)$ and $(d)$ match the X-point propagation and are being used to calculate the projection of the mode rotation velocity on poloidal direction.

the diamagnetic frequency (due to the electron diamagnetic current) $f_{\mathrm{e}}^{*}$ can be calculated for the time of the rotation inversion [21]:

$$
f_{\mathrm{MHD}}=n \frac{v_{\mathrm{tor}}}{2 \pi R_{0}}+m \frac{v_{\mathrm{pol}}}{2 \pi r}+m f_{\mathrm{e}}^{*}
$$

where $m$ and $n$ are the poloidal and toroidal mode numbers, $v_{\text {tor }}$ and $v_{\text {pol }}$ are the toroidal and poloidal ion velocities, respectively, and the sign depends on whether or not the toroidal magnetic field is oriented in the same direction as the plasma current. In equation (3.3.1), it has been assumed that 
the MHD eigenfrequency is negligibly small. The electron diamagnetic frequency is given by

$$
f_{\mathrm{e}}^{*}=\frac{1}{2 \pi e n_{\mathrm{e}} B_{\mathrm{t}}} \frac{1}{r} \frac{\partial p_{\mathrm{e}}}{\partial r} \approx \frac{1}{2 \pi e B_{\mathrm{t}} r} \frac{\partial T_{\mathrm{e}}}{\partial r},
$$

under the assumption that the electron density does not change too much in the vicinity of the $m / n=2 / 1$ island. The toroidal velocity, in its turn, consists of the components parallel and perpendicular to the magnetic field line, $v_{\text {tor }}^{\|}$and $v_{\text {tor }}^{\perp}$. This means that the ECE-imaging measurement of the rotating island is not able to distinguish between purely poloidal velocity component and the toroidal rotation velocity projected on the poloidal direction. As will be shown below, in some cases the poloidal rotation is quite essential and, therefore, cannot be neglected.

As has been described before, at $\sim 0.575 \mathrm{~s}$ the mode rotation direction has been reversed. At this moment, equation (3.3.1) can be rewritten as:

$$
\frac{1}{B_{\mathrm{t}}} \frac{\partial T_{\mathrm{e}}}{\partial r}=\left[\frac{n}{m} \frac{r}{R_{0}} v_{\mathrm{tor}}+v_{\mathrm{pol}}\right]
$$

From the CXRS diagnostic, one can derive a value for the ion toroidal rotation at $q=2\left(r_{q=2}=0.37 \mathrm{~m}\right)$ being $1.2 \times 10^{4} \mathrm{~m} \mathrm{~s}^{-1}$ (figure 7). The time averaged electron temperature gradient at $q=2$ is about $(1-1.5) \times$ $10^{3} \mathrm{eV} \mathrm{m}^{-1}$, yielding the ion poloidal rotation velocity $v_{\text {pol }}$ to be $\sim 580-850 \mathrm{~m} \mathrm{~s}^{-1}$.

According to the neoclassical theory [22,23], the poloidal rotation can be written as:

$$
v_{\mathrm{pol}}=5.33 \times 10^{-22} k \frac{c}{e B_{0}} \frac{\partial T_{\mathrm{i}}}{\partial r},
$$

in which $k$ is a numerical factor depending on the collisionality (in this case, $k \approx 0.4$ ), $c$ is the speed of light, and $\mathrm{d} T_{\mathrm{i}} / \mathrm{d} r$ is the ion temperature gradient in the $q=2$ vicinity. The CXRS measurements give the ion temperature gradient of about $3.4 \mathrm{keV} \mathrm{m}^{-1}$, leading to the poloidal velocity of $560 \mathrm{~m} \mathrm{~s}^{-1}$.

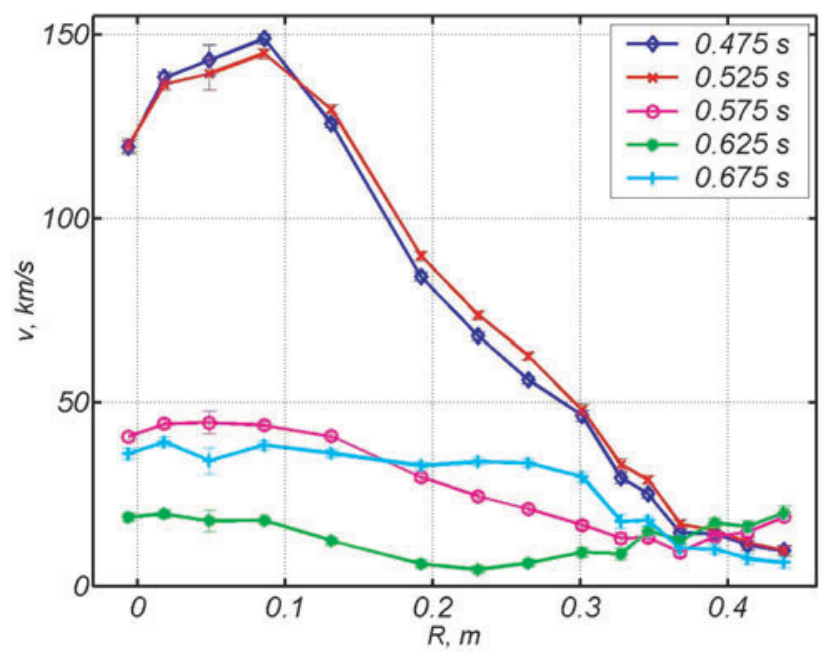

Figure 7. The ion velocity rotation profiles for the same shot as in figure 6. The sampling rate of the CXRS system is $50 \mathrm{~ms}$, meaning that fast change in the toroidal velocity cannot be observed.
This is equal to or less than the value derived from the experimental values put into equation (3.3.3).

It should be noted that in more standard TEXTOR discharges with strong momentum input given by NBI the toroidal plasma rotation of $10^{5} \mathrm{~m} \mathrm{~s}^{-1}$ dominates the observed MHD frequency in equation (3.3.1) with only minor contribution from the poloidal rotation and the diamagnetic frequency.

\subsection{Three-dimensional reconstruction of the large $m / n=2 / 1$ mode}

Using different ECE diagnostics, a three-dimensional mode reconstruction has been made for the same shot as in figure 2 . The evolution of the $m / n=2 / 1$ mode around the torus is shown in figure $8(a)$. The flat spot at the O-point is very pronounced, compare to the X-point. For this discharge, an 3/1-mode activity has been also detected (figure $8(b)$ ). The $q=3$ position is estimated to be at $r=0.43 \mathrm{~m}$. From the ECEimaging system, similar evolution in the poloidal direction is shown in figure $9(a)$. In figure $9(b)$ the temperature profiles from the vertically aligned ECE-imaging diagnostic show how the vertical position of the $\mathrm{X}$-point changes in time.

In the contour plot in figure 10 , for the radial set of ECE channels, the 'hot' spot of the $m / n=1 / 1$ mode is
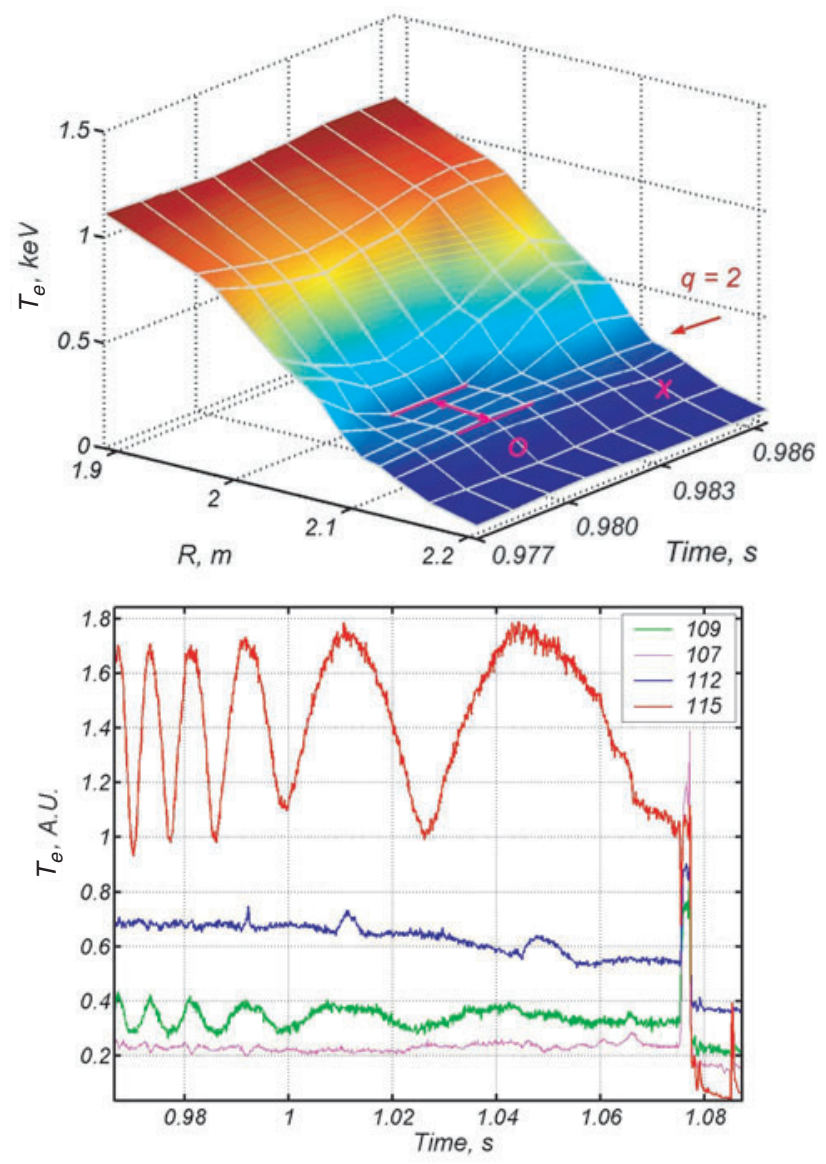

Figure 8. The three-dimensional mode reconstruction for one rotation period around the torus. The $\mathrm{O}$ - and $\mathrm{X}$-points of the $m / n=2 / 1$ island are clearly visible. In the bottom plot, channels at 109 and $107 \mathrm{GHz}$ indicate the presence of a 3/1 mode. 

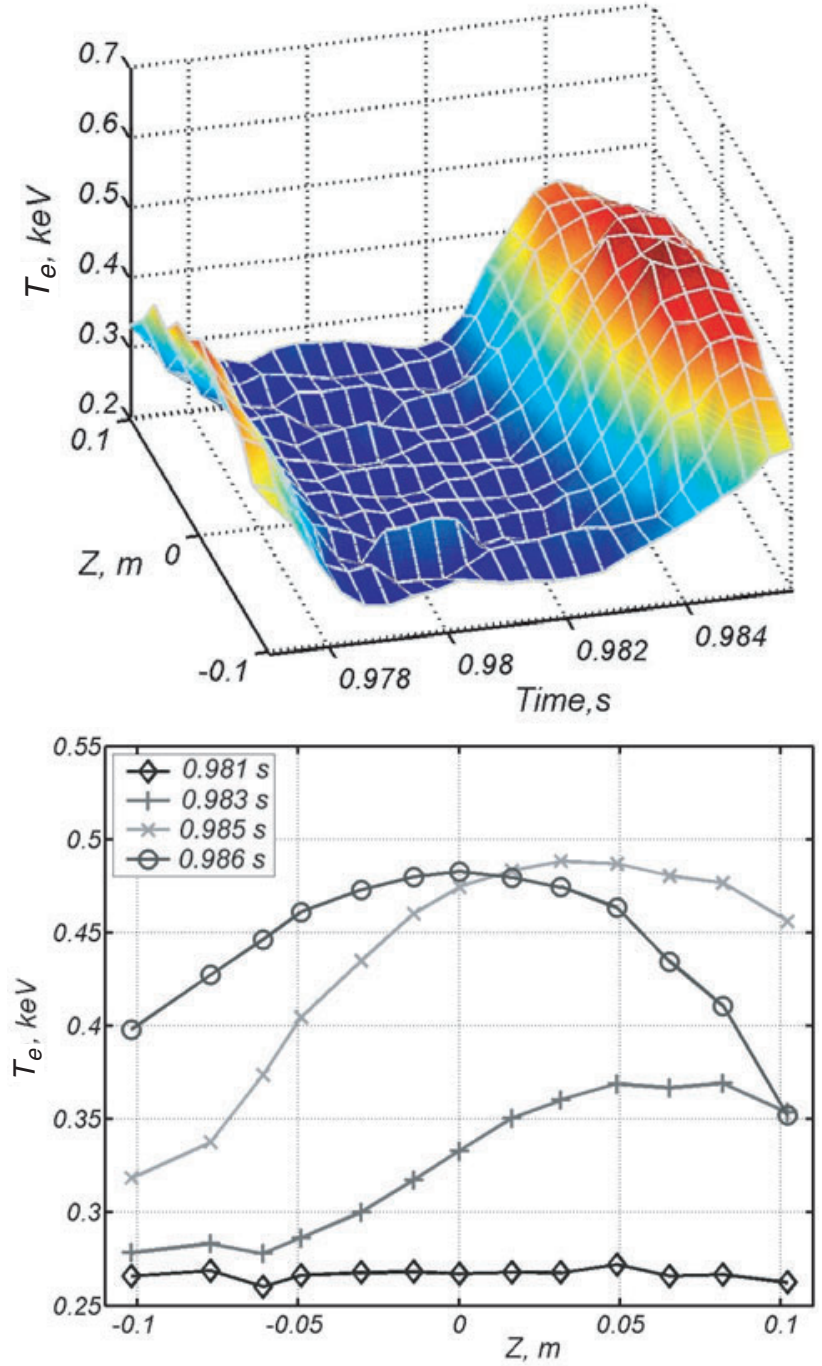

Figure 9. The three-dimensional reconstruction of the $m / n=2 / 1$ mode in the poloidal direction. The temperature profiles shows how the $\mathrm{X}$-point is moving through the viewline of the vertical set of ECE-imaging channels.

always in phase with the X-point of the $m / n=2 / 1$ island at the LFS. This has also been observed at RTP [24]. In this shot, no sawtooth activity has been detected, so this kind of $m=1$ island behaviour is typical of a slow precursor, when it couples with the $m=2$ and 3 modes further outwards. This behaviour leads to the disruption (minor or major). Some mechanisms that lead to the disruption through the coupling between different modes are described in [25]. Since the $m=1$ mode does not show a clear tearing mode topology in this shot, the terminology of 'hot' and 'cold' spots, instead of $\mathrm{X}$ - and O-points, will be used. It should be noted that the $m=1$ mode has a strong asymmetry with respect to the plasma centre at $R=1.82 \mathrm{~m}$, so its 'hot' spot is much more pronounced at the LFS than at the HFS. Both this and the radial $m / n=2 / 1$ island asymmetries are an indication that ballooning effects are taking place.

From figures $8-10$, the full picture of the island phases at TEXTOR can be made. Figure 11 shows the mode topology for the phases when the $\mathrm{O}$ - and X-points, respectively, are located in the equatorial plane at the LFS.

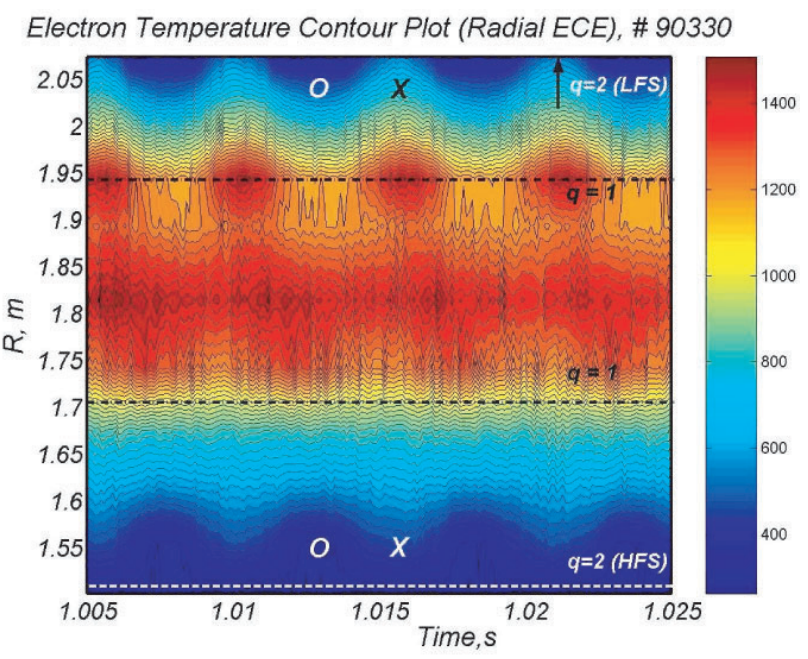

Figure 10. Evolution of the $m=2$ and 1 modes as seen by the radial set of the ECE channels. The central $m=1$ mode is coupled to the $m=2$ mode such that its 'hot' spot is always in phase with the X-point at the LFS
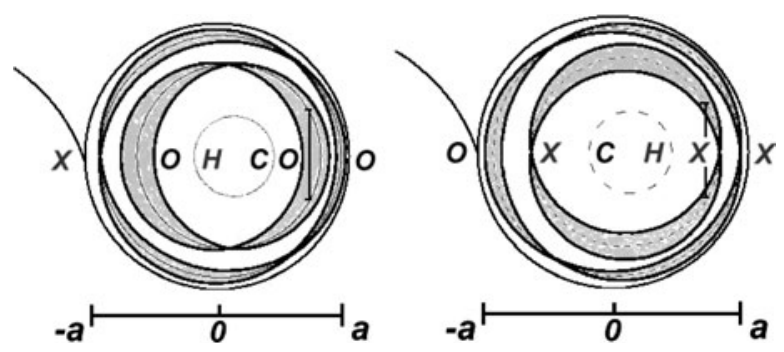

Figure 11. Schematic drawing of the relative phases of the islands with different $m$ numbers. The ECE-imaging line of sight is shown at the LFS.

\subsection{Evidence of a small-scale mode with higher m number associated with the large $m=2$ island}

One of the new results obtained recently with the ECE diagnostics at TEXTOR is the evidence of a small-scale mode associated with the large $m=2$ island. In figure 12, a periodic increase of the temperature on top of the main $m=2$ mode is seen by the fast ECE spectrometers at the HFS and by the 11-channel ECE radiometer at the LFS. Earlier, the same behaviour has been observed by the pulsed radar reflectometer in the density time traces [5]. In these shots, co- and counterNBI with the same powers has been used. To make a proper estimation of $m$ and $n$ numbers for this mode, its location should be identified first. The $q=2$ position is found to be at $R=1.52 \mathrm{~m}$ at the HFS and at $R=2.11 \mathrm{~m}$ at the LFS. It should be noted that the small mode has appeared immediately after the minor disruption, and no traces of this mode have been found before it. Some sawtooth activity has been detected just after the minor disruption for about $6 \mathrm{~ms}$, but then it has vanished (figure 13(a)), and an $m=1$ mode develops with the same characteristics as described earlier in section 3.4. Suddenly, the amplitude of the large $m / n=2 / 1$ island is modulated by the small mode. Analysis shows that the frequency of the small mode is twice as much as that for the $m / n=2 / 1$ island and equals $1.3 \mathrm{kHz}$ after the disruption (figure 13(b)). This is also in agreement with the measurements 

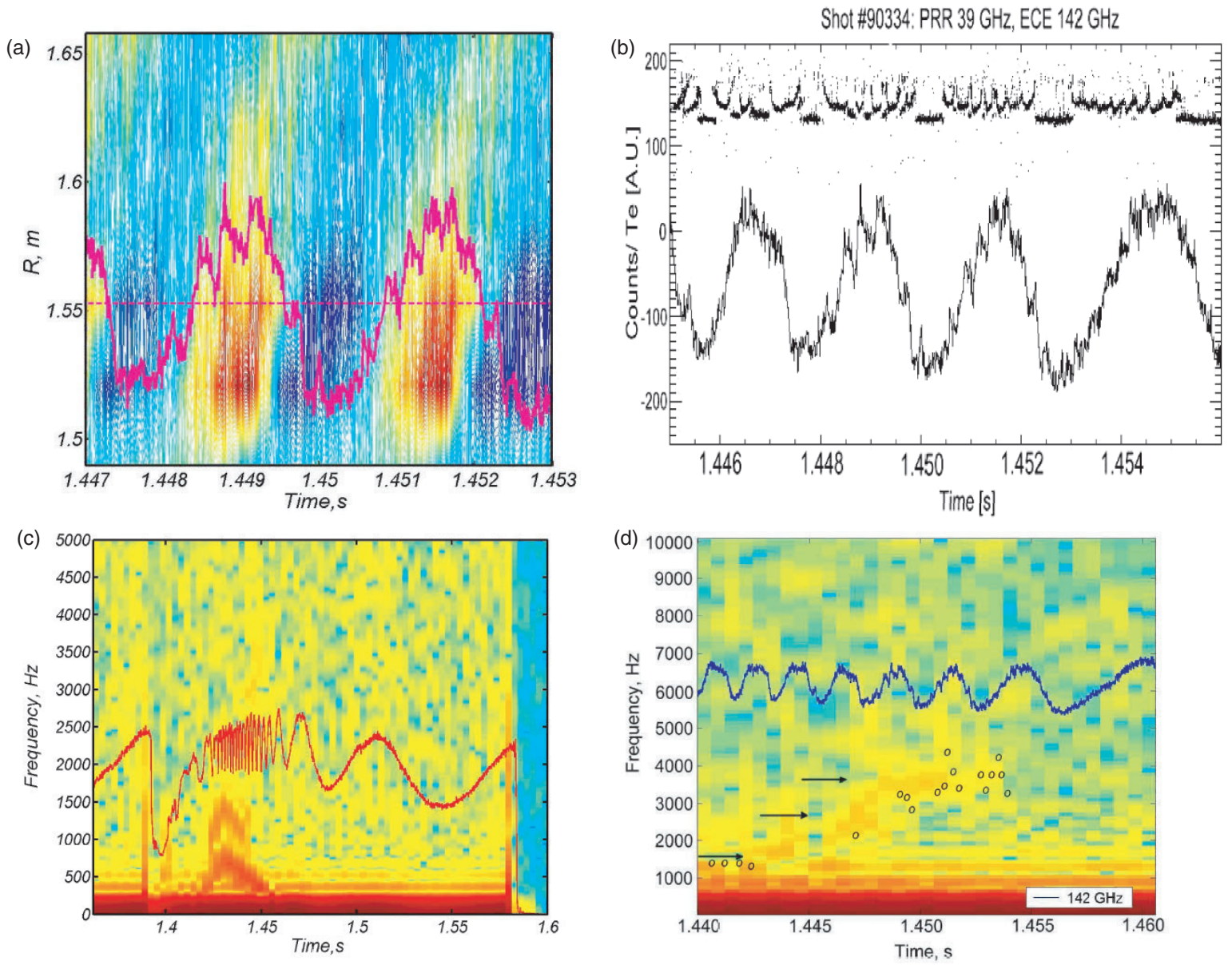

Figure 12. Evidence for a small mode in TEXTOR: $(a)$ the contour plot for $\left(T_{\mathrm{e}}-\bar{T}_{\mathrm{e}}\right) / \bar{T}_{\mathrm{e}}$ shows periodical increase of temperature on top of the $m=2$ mode; $(b)$ pulsed radar reflectometer (top) and ECE (bottom) raw signals clearly indicate the fast change in frequency for the high $m$ modes between 1.447 and $1.455 \mathrm{~s} ;(c)$ spectrogram of the LFS ECE signal $(107 \mathrm{GHz}) ;(d)$ spectrogram of the HFS ECE signal (142 GHz) with arrows showing the frequency change of the small-scale mode from 1.5 to $3.7 \mathrm{kHz}$, dots are results obtained with the help of a pulsed radar reflectometer.

performed by magnetic diagnostics, which show the presence of a mode with $n=2$. The small-scale mode remains coupled with $m=1$ and 2 modes up to a certain time, and then its frequency suddenly increases from 1.5 to $3.7 \mathrm{kHz}$ in a very short time interval of about $7 \mathrm{~ms}$. At the same time, the $m=1$ and 2 modes are still coupled and continue to slow down. There are two possibilities that could possibly lead to this kind of behaviour:

- The observed small mode could be a $3 / 2$ mode. From figure 5(a), a modulation caused by this mode is pronounced inside the $q=2$ surface. From the ECE data, the location of $q=1.5$ is estimated to be somewhere between $R=1.97-2.04 \mathrm{~m}$ at the LFS and $R=1.56-1.62 \mathrm{~m}$ at the HFS. The uncertainty of $6-7 \mathrm{~cm}$ is due to the large radial separation between the channels at this radial position. However, the mechanisms that force the $m / n \approx 3 / 2$ mode to uncouple at some time from the strongly coupled $m=1$ and 2 modes are not very clear. From the CXRS measurements, it follows that the toroidal ion velocity is very small at values of $3-7 \mathrm{~km} \mathrm{~s}^{-1}$ in the vicinity of $q=3 / 2$. This means that differences in the diamagnetic frequency and/or the poloidal ion velocity can create a large difference in $f_{\mathrm{MHD}}$ of the various modes.
Thus, a sudden change in the local diamagnetic pressure could possibly cause the small mode to uncouple from the $2 / 1$ and $1 / 1$ ones.

- A higher- $m$ mode with $1<m / n<2$. The break-up of the magnetic topology could lead to the creation of a chain of small islands with higher $m$ and $n$ numbers (e.g. with $m=6-12$ and $n=4-8$, respectively) but with their ratio $1<m / n<2$. With the present spatial resolution of the ECE diagnostics this possibility could not be further investigated.

\section{Temperature fluctuations associated with the large MHD modes}

As has been shown earlier in [26], the broadband density fluctuations in the presence of the large $m=2$ mode at TEXTOR are enhanced at the X-point of the island, compared to its O-point (see also figure 14). To measure the temperature fluctuations, a cross-correlation analysis between neighbouring ECE-imaging channels has been done. When frequency bands are very close, they correspond to mostly overlapped sample volumes, so that r.m.s. value and cross power spectrum of the local $T_{\mathrm{e}}$ fluctuations can be calculated 

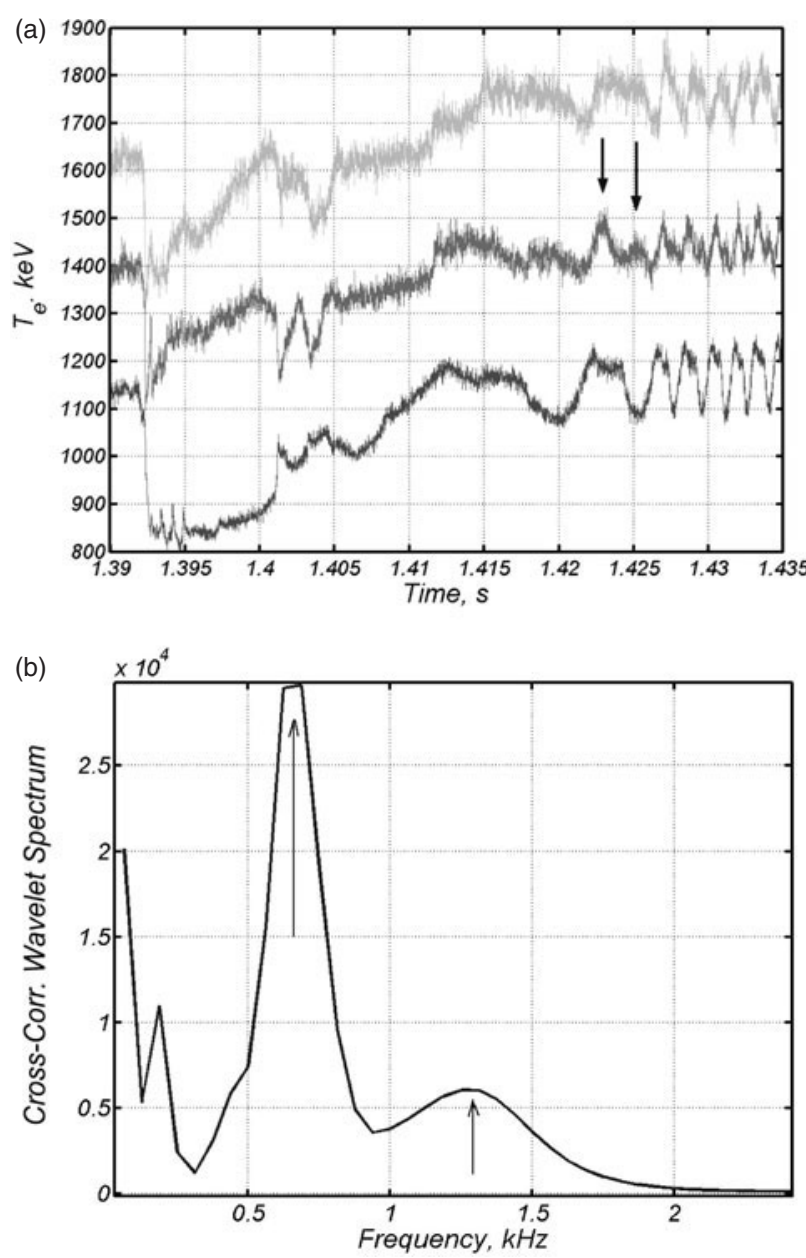

Figure 13. The behaviour of the electron temperature as measured by ECE after a minor disruption ( $a$ ) at $q=1.5$. The mode with $n=2$ can be seen soon after $1.42 \mathrm{~s}$ as a modulation on top of $2 / 1$ mode oscillations and indicated by arrows. It is coupled to the $m=2$ mode and has a twice as high frequency of $1.3 \mathrm{kHz}$, as can be seen from the cross-correlation wavelet spectrum obtained at the period from 1.42 to $1.435 \mathrm{~s}(b)$

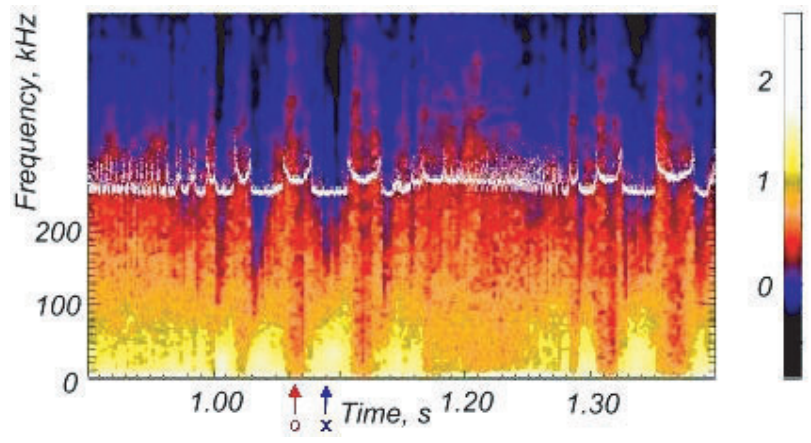

Figure 14. Power spectra of a pulsed radar reflectometer channel (33 GHz, LFS) shows an enhancement of density fluctuations below $\sim 100 \mathrm{kHz}$ at the $\mathrm{X}$-point of the $m / n=2 / 1$ island, compare to the O-point [26]. In this figure, light colour corresponds to a higher power level.

from the cross-correlation between these signals. These adjacent channels are slightly displaced above and below the midplane on the LFS. The principles of the temperature fluctuation measurements by this method are described in detail in [27,28]. For this analysis, the time interval of one mode oscillation (figure 15(a)) for the same shot as in figure 2 has been subdivided into several windows. The mean temperature was subtracted from the signals and they were subsequently subjected to a wavelet transform, which rather than a Fourier transform, gives better accuracy for the relatively short data records and does not average the spatial information [29]. One should note, however, that these time intervals are very short, so the accuracy in determination of exact fluctuation frequencies and amplitudes is rather high even for the wavelet transform, so mainly a qualitative analysis can be made. These observations are in agreement with those for density fluctuations. The wavelet cross-spectrum shows an enhancement of the broadband temperature fluctuations in the vicinity of the X-point, compared to the O-point of the $m=2$ island (figure 15(b)). It is necessary to mention, however, that the r.m.s. values of the fluctuations, normalized to the mean value of the temperature for each time window (figures $15(d)$ and (e), see the method described in [30]), as well as their spectra, do not show a significant difference between $\mathrm{O}$ - and $\mathrm{X}$-points. If one uses a mixing length argument, one derives for $\tilde{T}_{\mathrm{e}} / \nabla T_{\mathrm{e}}$ a length scale of $1 \mathrm{~cm}$ at the X-point and $4 \mathrm{~cm}$ at the O-point. In conclusion, one can say that the turbulence characteristics are quite different: at the X-point small-scale lengths at high frequencies; at the $\mathrm{O}$-point long-scale lengths at low frequencies. The detailed studies of the broadband temperature fluctuations under different plasma parameters and heating regimes will be done in future, when a dedicated ECE correlation technique will become available.

\section{Conclusion}

Comparison between two models for the plasma transport properties study inside the large MHD islands gives estimations of the heat diffusivities which are much lower than the global plasma heat diffusivity. The three-dimensional reconstruction of large modes in TEXTOR allows to model the island as a structure with closed flux surfaces. The main plasma heat flux flows through the X-point area, probably along stochastic magnetic field lines. The perpendicular transport through the island is very much reduced. This is confirmed by a temperature profile flattening and even secondary peaking inside the island, compared to the X-point. The same observations have been made in the electron density profiles. The broadband temperature and density fluctuation measurements in the vicinity of the X-point confirm that the magnetic topology is probably stochastic there, compared to the O-point.

The mode rotation studies with the ECE-imaging diagnostic have shown that the purely poloidal component of plasma rotation can be significant in some Ohmic and NBI-heated discharges at TEXTOR, especially when large modes are present with low toroidal ion velocity. It is confirmed that if the $m=1,2$ and 3 modes with $n=1$ number are coupled, they have their X- or O-points in phase at the LFS. The large islands are almost always asymmetric at the LFS and symmetric at the HFS, although, the total island width is the same in both cases.

Both the ECE and the pulsed radar reflectometer diagnostics have confirmed an evidence of a small mode with 

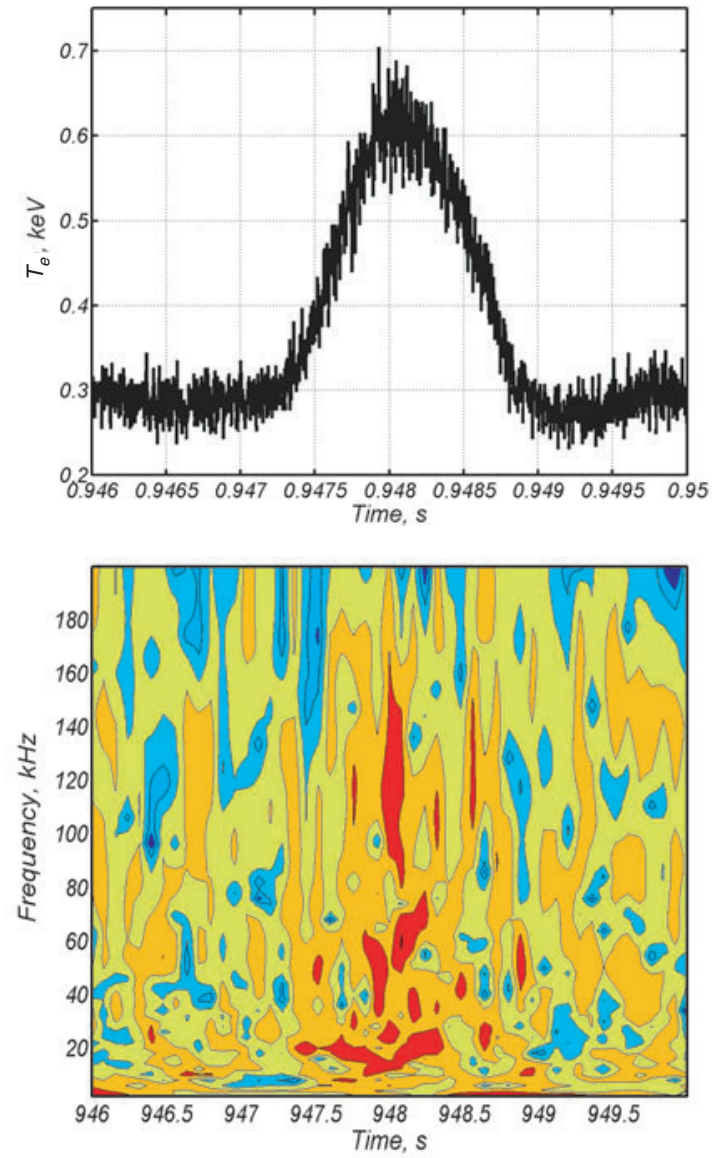
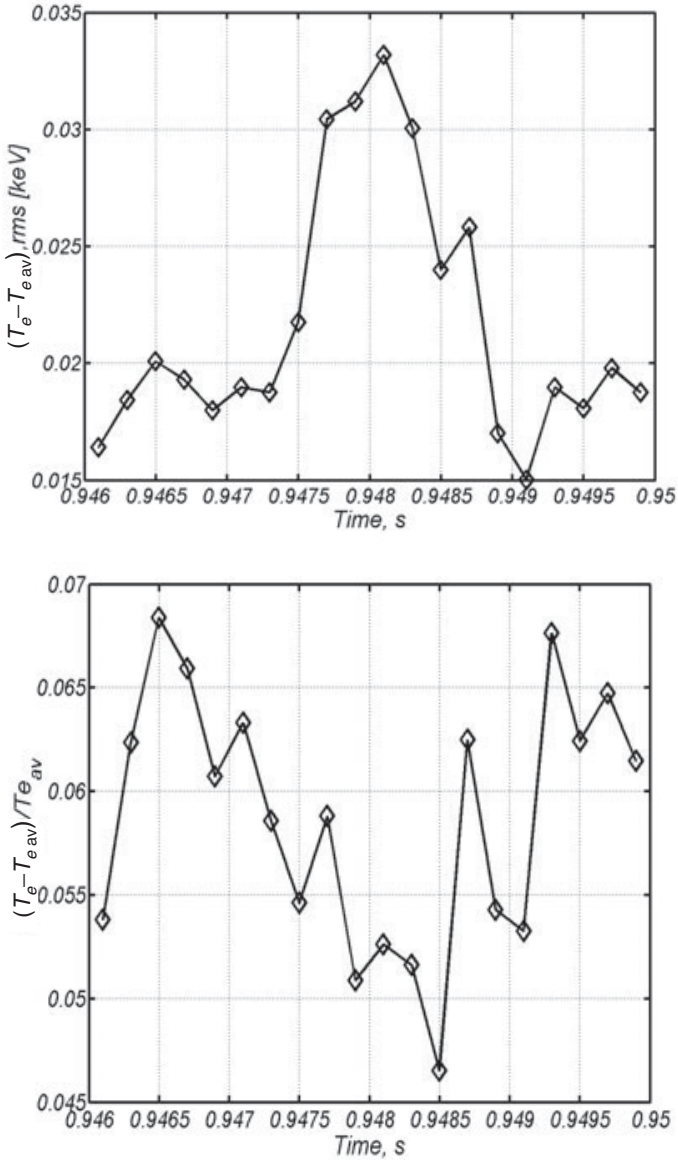

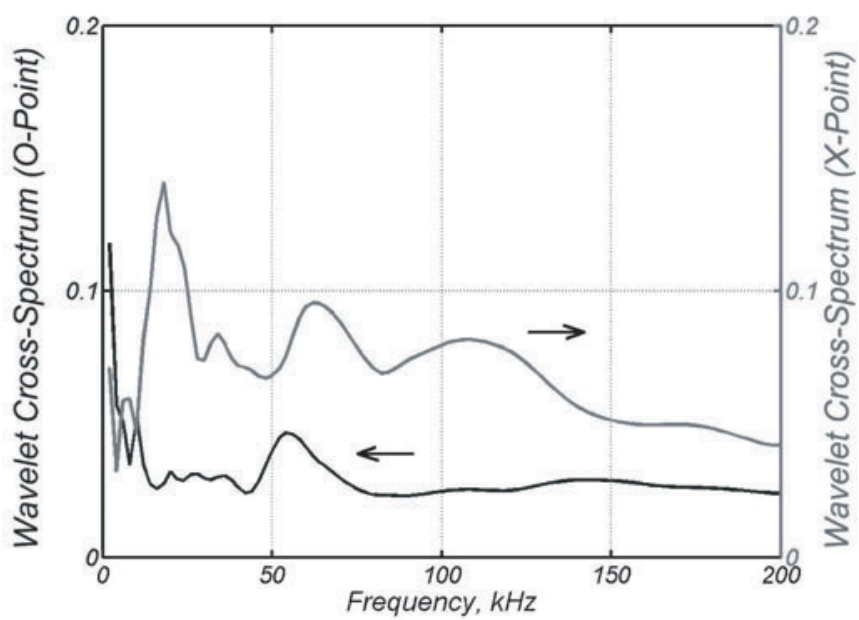

Figure 15. The (wavelet) cross-spectrum $((b)$, dark colour corresponds to a higher power) of two adjacent ECE-imaging signals $((a)$, only one signal is shown) shows an enhancement of the temperature fluctuations in the X-point vicinity of a large $m=2$ mode. Wavelet power spectra for the $\mathrm{O}$ - and the X-points $(e)$ shows that these fluctuations are in the frequency range of $20-150 \mathrm{kHz}$, though higher frequencies dominate the turbulence characteristics for the X-point, compared to the O-point. The r.m.s. values for the mean-removed signal (c), as well as the normalized r.m.s. values $(d)$, are given for comparison.

$1<m / n<2$. The mechanisms that force this mode to uncouple from the $1 / 1$ and $2 / 1$ modes are not very clear yet.

\section{Acknowledgments}

The authors would like to thank Dr R. Jaspers for the useful discussions and help with the CXRS diagnostic data,
Dr W. Biel for his help in analysis of the VUV/XUV spectrometer data, and Dr D. Hogeweij for the help with calculation of the current density profile in the neoclassical approach.

This work was performed under the EURATOMFOM association agreement with financial support by the 'Nederlandse Organisatie voor Wetenschappelijk Onderzoek 
(NWO)', the 'Stichting voor Fundamenteel Onderzoek der Materie (FOM)', EURATOM and Forschungszentrum Jülich $\mathrm{GmbH}$.

\section{References}

[1] de Vries P.C. et al 1997 Nucl. Fusion 371641

[2] Krämer-Flecken A. et al 2003 Nucl. Fusion 431434

[3] Rutherford P.H. 1985 PPPL Report 2277

[4] van Milligen B.Ph. et al 1993 Nucl. Fusion 331119

[5] van Gorkom J.C. et al 2001 Proc. 5th Int. Reflectometry Workshop (Toki, Japan, 2001) NIFS Research Report NIFS-PROC-49 pp 59-63

[6] Udintsev V.S. et al 2002 Proc. 12th Joint Workshop on ECE and ECRH (Aix-en-Provence, 13-16 May 2002) ed G Giruzzi (Singapore: World Scientific) p 215

[7] Udintsev V.S. et al 2001 Rev. Sci. Instrum. 72359

[8] Donné A.J.H. et al 2001 Rev. Sci. Instrum. 721046

[9] Deng B.H. et al 2001 Rev. Sci. Instrum. 72368

[10] Barth C.J. et al 2001 Rev. Sci. Instrum. 721138

[11] van de Pol M.J. et al 2002 Proc. 12th Joint Workshop on ECE and ECRH (Aix-en-Provence, 13-16 May 2002) ed G Giruzzi (Singapore: World Scientific) p 203

[12] van Gorkom J.C. et al 2001 Rev. Sci. Instrum. 72336
[13] Fitzpatrick R. 1995 Phys. Plasmas 2825

[14] Salzedas F. et al 2002 Phys. Rev. Lett. 88075002

[15] Soltwisch H. 1992 Plasma Phys. Control. Fusion 341669

[16] Spitzer L. and Härm R. 1953 Phys. Rev. 89977

[17] Rapp J. et al 1999 Forschungszentrum Jülich Report Jül 3697

[18] Udintsev V.S. et al 2000 Proc. 27th EPS Conf. on Control. Fusion and Plasma Phys. (Budapest, Hungary) vol 24B (ECA) p 948

[19] van Milligen B.Ph. et al 2002 Rev. Sci. Instrum. 743998

[20] White R.B. and Monticello D.A. 1997 Phys. Fluids 20800

[21] de Vries P.C. et al 1997 Plasma Phys. Control. Fusion 39439

[22] Hazeltine R.D. 1974 Phys. Fluids 17966

[23] Bugarya V.I. et al 1985 Nucl. Fusion 251707

[24] Salzedas F.J.B. 2000 The disruptive instability in tokamak plasmas PhD Thesis University of Utrecht

[25] Mirnov S.V. et al 1998 Phys. Plasmas 53950

[26] van Gorkom J.C. et al 2001 Proc. 28th EPS Conf. on Control. Fusion and Plasma Phys. (Funchal, Portugal) vol 25A (ECA) p 1401

[27] Cima G. et al 1995 Phys. Plasmas 2720

[28] Deng B.H. et al 2001 Rev. Sci. Instrum. 72301

[29] van Milligen B.Ph. et al 2001 Nucl. Fusion 41447

[30] Herranz J. et al 2000 Phys. Rev. Lett. 854715 